\title{
Rethinking health services measurement for Indigenous populations
}

\author{
Angela Mashford-Pringle ${ }^{\mathrm{a}, *}$, Ian Ring ${ }^{\mathrm{b}}$, Fadwa Al-Yaman ${ }^{\mathrm{c}}$, John Waldon ${ }^{\mathrm{d}}$ and Michelle Chino ${ }^{\mathrm{e}}$ \\ ${ }^{a}$ Waakebiness-Bryce Institute for Indigenous Health, Dalla Lana School of Public Health, University of Toronto, \\ Toronto, ON, Canada \\ ${ }^{\mathrm{b}}$ Research and Innovation Division, University of Wollongong, NSW, Australia \\ ${ }^{\mathrm{c}}$ Indigenous and Maternal Health Group, Australian Institute of Health and Welfare, ACT, Australia \\ ${ }^{\mathrm{d}}$ School of Public Health and Community Medicine, UNSW, Sydney, Australia \\ ${ }^{\text {e }}$ School of Community Health Sciences, University of Nevada, Las Vegas, NV, USA
}

\begin{abstract}
Indigenous people around the world experience shorter life expectancy, poorer health outcomes, and on average have less social capital, than non-Indigenous people in their respective countries. While national goals are to lower mortality and morbidity rates of Indigenous people, much evidence exists that indicates there is almost no Indigenous involvement in data collection, policy development, program implementation and development and measurement of services. A more holistic and culturally relevant framework is presented to improve services and outcomes for Indigenous populations.
\end{abstract}

Keywords: Indigenous people, health services, population health, Global Indigenous health, changing health statistics, Indigenous epidemiology

\section{Introduction}

At a meeting of the International Group for Indigenous Health Measurement (IGIHM) in Atlanta, Georgia in November 2017, scholars from around the world discussed many issues related to Indigenous health. It was recognized at this meeting that more must be done to move towards Indigenous people-led service agendas. The meeting participants from Australia, Canada, New Zealand and the United States concluded that data must encompass Indigenous ways of knowing and being and the impact of colonisations that include disruption to family, culture and spirituality, and relationships with land which are not currently captured by the epidemiological statistics. Further, national/regional/state and local statistical agencies and health policy makers

\footnotetext{
*Corresponding author: Angela Mashford-Pringle, WaakebinessBryce Institute for Indigenous Health, Dalla Lana School of Public Health, University of Toronto, 155 College Street, Room 404, Toronto, ON, Canada. Tel.: +1 416978 8771; E-mail: angela. mashford.pringle@utoronto.ca.
}

must work together with their Indigenous counterparts to ensure health data reflect specific prevention, service utilization, and outcome indicators relevant to Indigenous communities. This paper presents a holistic framework for the measurement of culturally appropriate services for Indigenous people that was discussed at this meeting.

\section{Background}

A review of health statistics illustrates the large gaps in health status between non-Indigenous and Indigenous peoples in Australia, Canada, New Zealand and the United States [1,2]. Health outcomes are determined by the characteristics of the population including their composition, socioeconomic status, health needs and health risk factors; characteristics of the area in which they live including community connectedness/safety, housing stock, employment opportunities, transport and remoteness. The availability, accessibility, effectiveness and cultural appropriateness of health 
services is also a key determinant of health outcomes. In all the four countries Indigenous people have access to Indigenous specific primary health care services but can also access services available to the nonIndigenous population (called main stream primary health care services). The relative contribution of Indigenous specific services relative to main stream services is different in the four countries. As such it is difficult to compare their impact on the overall health status and health outcomes. In addition, many of the contextual factors pertaining to emotional, social, economic, geographic, and political issues that greatly contribute to this health gap are measured to a varying extent in different countries. Some countries attempt to measure the personal values and perceptions of Indigenous people. For example, in Australia, significant efforts have been made to measure social and emotional well-being as well as to examine the contribution of social determinants of health to health outcomes including life expectancy [3-7]. In the US, the 30year Healthy People Initiative has expanded the ability to include social determinants in national, tribal, state, and local health measurement and in 2011, the US Surgeon General's National Prevention Strategy included the need to standardize and collect data to better identify and address disparities, specifically including tribal governments [8]. In Canada, First Nations Information Governance Centre (FNIGC) works with First Nations communities across Canada to conduct, analyze and report on health and contextual issues (see First Nations Regional Health Survey 2016) [9].

The historical contexts that lie behind the poor health outcomes of Indigenous people in Australia, Canada, New Zealand and the United States are not represented in the epidemiological statistics. The numbers generally do not recognise causes like intergenerational trauma affecting Indigenous people as a result of forced removal of children from family (Stolen Generations, Residential Schools, Boarding Schools, Sixties Scoop and negative child welfare policies), separation from traditional homelands (forced removals, unbalanced and unfulfilled treaties, continued intrusion into lands reserved for Indigenous people), and forced assimilation [10-12]. Studies in Canada [13], Australia [14,15], and the United States [16] illustrate the intergenerational trauma of removing Indigenous children from their families. Further, national health surveillance systems in the US, such as the Centers for Disease Control's Behavioral Risk Factors Surveillance System (BRFSS), are increasingly including social context indicators and providing sup- port for Indigenous peoples to modify the base survey to include indicators relevant to them [17]. There is a Tribal BRFSS Toolkit that provides examples of how tribal communities can use BRFSS survey in American Indians/Alaskan Natives (AI/AN) populations, which would allow the inclusion of tribal-specific questions to assist in the measurements of health risks associated with AI/AN populations and how culturally appropriate and safe interventions are impacting these health risks. The BRFSS survey has core and optional modules that can be administered through random sampling of AI/AN peoples [18]. The findings from these studies provide much needed contextual information, that when combined with quantitative health measurements, could lead to culturally and linguistically appropriate Indigenous health and social services that are led or co-led by Indigenous peoples. Another example of contextualization of data was demonstrated in the Chandler and Lalonde study in Canada [18]. They hypothesized that when an Indigenous community had high levels of 'cultural continuity', there was a reduction in the number of youth suicides in First Nations communities in Canada. They stated that cultural continuity was characterized as people who had connection to cultural events and activities (i.e., hunting, fishing, ceremonies, singing, dancing, storytelling, healing and traditional medicine), knowledge of or use of an Indigenous language, and a sense of belonging to and a positive sense of identification with, an Indigenous community. Such things have been neglected in commonly used statistical measures.

Isolation and stigmatisation in mainstream society and services impact adversely on Indigenous populations. These circumstances need to be urgently addressed with a range of appropriate services. Routine statistics have not consistently considered the social, emotional, and spiritual causes of ill health, in either Indigenous or non-Indigenous people. Health programs continue to focus predominantly on an 'illness/treatment' paradigm and on a medical model when that may not be the only or optimal solution. Rather than medication or hospitalization as the only response to illness, a holistic approach that examines why an individual has presented to a health professional seeking care, may be more appropriate [19].

Until very recently, only biomedical models of health care have been funded. Indigenous healing, traditional medicines and ways of knowing are not funded because they have not been subjected to the positivistic and randomised controlled trials research that is medical science. Indigenous healing considers the physical, 
social, emotional, spiritual, and economic aspects of a person's life in relation to their illness. Biomedicine examines illnesses in isolation from the social and economic background of the patient. Indigenous scholars have argued that Indigenous healing practices and traditional medicines should also be the subject of medical research [20-22]. Successful, albeit rare models exist, for services that combine Indigenous and mainstream healing. In the US, for example, the Indian Health Service (IHS) has funded programs that combine accepted native traditional medicine and modern medical technology $[23,24] .{ }^{1}$ But the lack of standard indicators for measuring Native medicine and $\mathrm{Na}-$ tive health prevents more widespread adoption of these combined approaches. In this context, it is worth noting the World Health Organization will be releasing the new International Classification of Diseases (ICD-11) in May 2019, which will have a chapter on traditional medicines.

\section{A best practice framework Indigenous health services}

Changes are being made to the health systems in all four countries that are the subject of this paper, but substantive changes that examine more than the absence of disease need to be introduced for the benefit of Indigenous peoples. Moving away from a framework that focuses on measuring disease prevalence, morbidity and mortality to a framework that focuses on Indigenous wellness or wellbeing was also discussed at the Atlanta meeting and is seen as a positive way forward to focus on elements that make Indigenous people well. In fact, a range of Indigenous wellness and wellbeing frameworks are currently being developed in the four countries. Leininger's [25] transcultural nursing theory or culture care theory states that health care providers must tailor their care to fit with the individual, family, group or institution's cultural values, beliefs and ways of life, in order to provide appropriate and culturally-informed care that contributes to improved health and well-being. An examination of Indigenous health and well-being in the countries of interest, finds that few health professionals see the need to be culturally sensitive and aware of different cultural

\footnotetext{
${ }^{1}$ Support for these initiatives and for increasing tribal ownership of their own health services has come from two key pieces of US national legislation - the 1975 Indian Self Determination Act and the 1978 American Indian Religious Freedom Act.
}

differences when providing care, or to integrate models of holistic care. To facilitate change, there needs to be a focus on the kind and quality of health statistics used to design and fund health services, as well as mechanisms to support partnerships between governments and Indigenous populations.

For Indigenous health systems to succeed, Indigenous people and their communities must be part of the discussions that lead to policy, program development and service delivery at local levels. The incorporation into the health system of culture, language, and/or ceremonies should take place, as needed or desired by Indigenous communities. The organization and delivery of health care services could better meet the needs of Indigenous people and their communities by the inclusion of a wide variety of practices, including the acceptance of cultural beliefs and traditional healing, if communities desire that. In all four countries, Indigenous people argue that culture should be at the center of any work relating to elimination of health disparities such as Australia's Closing the Gap Refresh agenda [30]. There are a range of strategies to ensure that this is implemented across the four countries. These including cultural awareness training, cultural competency training and cultural respect and safety training. The latter places a strong focus on non-Indigenous people in terms of reflecting on their values, culture and place in society, and how that impacts on their relationships with Indigenous Australians and the services they provide to them. The focus is on systems, racism, power and privilege. The US's Healthy People 2020 initiative seeks culturally relevant and safe health services.

It has been recognised that one of the main issues affecting access of Indigenous people to health services in all four of the countries included in this discussion, is a lack of cultural competency and an understanding of Indigenous cultures. Across our countries, health professionals are now provided with opportunities to complete cultural competency training that includes an understanding of the socio-political history of Indigenous people as well as an understanding of how their own cultural views hinders their capacity to treat Indigenous people. Recently Australian Health Ministers endorsed a Cultural Respect Framework that is going to be implemented across the health system [26] and supported by the New Zealand Medical Council as a prerequisite for good care and part of their statutory function under the New Zealand Health Practitioners Competence Assurance Act 2003 [27]. Health professionals who understand social, political, economic and historic issues facing Indigenous people can provide 
more empathetic and culturally safe programs and services, which will lead to improved health outcomes. However, often health professionals do not have the required cultural understanding. As a result, the interactions they have with Indigenous patients can influence when, how and from whom Indigenous people seek health services [28,29].

To move toward a more holistic and Indigenousfriendly health care model, it is important to identify which components of the current system will be required to change. A complete review of the health systems in Australia, Canada, New Zealand and the United States for Indigenous people would be a first step in creating a health systems framework that is holistic and includes the social determinants of health. A review of the health system needs to provide policy makers and program developers with an overview of preventable admissions to hospitals and preventable deaths, the use of specific services and their patterns of use. Regional, provincial and local level statistics can identify areas of chronic disease, common illnesses or socio-economic issues that need to be addressed in collaboration with Indigenous leaders and health and social services professionals. At the local level, statistics can help Indigenous health providers to identify areas that need to be funded and that require additional human resources, or other forms of support, to improve health outcomes.

In all four countries, governments rely on quantitative data derived from access and use of health care services to determine the delivery of services. However, not all countries have health statistics that consistently and reliably identify ethnicity to quantify what the needs are for their Indigenous populations. For example, in Canada, different provinces have different requirements for registering births such that some provinces and territories do not require or request racial or ethnic information, which then affects the type of data that is available for improving or changing programs and services related to infant and maternal health $[30,31]$.

The Indigenous populations of Australia, Canada, New Zealand and the United States are younger than the non-Indigenous populations. However, policy development, funding and services implementation for Indigenous people do not necessarily reflect this situation because the broader population is aging and do not share the same level of need of maternal/child services as the younger Indigenous population. It is imperative that contextual information be provided for policy makers to ensure that the needs of Indigenous people are recognized and the services they require are available to reduce the health outcome inequalities.

At the IGIHM meeting in Atlanta, six speakers ranged across many topics, including using maps in Australia to identify low-service areas, self-determination and self-governance in Canada, mental health and restorative justice in New Zealand, and facilities and childhood mortality in the United States. A common theme was that while health statistics can identify service gaps, more attention needs to be paid to the next step of planning and delivering services to close health outcome gaps. As such, essential components that need to be examined are outlined in the Framework (see Table 1), that included the measurement aspects of general services need, access and preference, requirements of specific service types, expenditure, workforce, social determinants including culture and racism, evaluation, trajectories and timing and community determination and empowerment. The framework focused on the implementation of a culturally competent, respectful holistic health system that includes Indigenous perspectives and views, which should drive data collection and data analysis.

The Framework for the Health and Wellbeing Services Measurement for Indigenous populations highlights ten key areas where local, regional, and national statistics should be used to create systemic change in health policy, programs and services. The Framework is populated with data that correctly and accurately records the ethnicity of the patient and their preferences for care, while considering their needs in a holistic manner. Access to appropriate and safe health care requires meeting the patients' needs in a manner that supports their humanity and dignity while being culturally safe and relevant. The data used must be standardized and of high quality so that it can be shared by organizations and governments who are working toward improving the health and wellbeing of Indigenous people. The last element of the framework is about integrating Indigenous self-determination into policy and practice at all levels of society to ensure informed decision-making, and ultimately, a set of foundational principles and commonly agreed-upon indicators are adopted. The Framework is intended to be a starting point to ensuring appropriate data is collected with collaboration of Indigenous people.

\section{Discussion}

The Framework for the Health Services Measurement for Indigenous Populations focuses on the re- 
Table 1

Framework for the health and wellbeing services measurement for Indigenous population

1. General services need (national and state/regional/provincial and local level of analysis)

(a) Preventable admissions and deaths (PAs/PDs)

(b) Current use of services in relation to service needs

(c) PAs/PDs in relation to use of services to identify areas with possible service gaps

2. Services availability and access

(a) Availability of and access to Indigenous controlled health services and mainstream population services on a population basis

(b) Individual/community preference and choice for Indigenous and/or mainstream service

(c) Barriers to access such as cost/distance and transportation/cultural appropriateness/lack of trust/limited services

(d) Involvement of Indigenous peoples in policy development, program design and implementation

3. Distribution of specific services relative to population and outcome indicators

(a) Maternal/child health: low birth weight and perinatal deaths, access to traditional birthing methods, antenatal attendance in total and in the first three months, maternal smoking cessation, substance/alcohol harm reduction, etc.

(b) Chronic disease: e.g., proportion of population with blood pressure, HbA1c, renal testing, percentage of undiagnosed hypertension, renal disease, diabetes, etc.

(c) Mental health and social/emotional well-being: culturally appropriate and responsive mental wellness services including crisis response and suicide prevention

(d) Health promotion by type: e.g. nutrition, smoking, alcohol and substance misuse, exercise, immunization, violence prevention including shelter services

(e) Environmental: clean water supply, sanitation, food supply, air quality

(f) Long term care, elder care

(g) Traditional healing and medicines use/practice

4. Services preference and choice

(a) Indigenous community controlled health services and mainstream health services

5. Human resources/workforce

(a) Indigenous/non-Indigenous health services workforce in relation to service need

(b) Indigenous/non-Indigenous health workforce by category (i.e., doctors, nurses, allied health professionals, dentists, etc.)

(c) Provider training in culturally appropriate, respectful and safe services - beyond bio-medical/health services training for all health professionals

(d) Workforce planning and educational pipelines for Indigenous students

6. Expenditures: per capita expenditures on health services in relation to need captured by total and by service type and health services including comparisons between Indigenous and total population per capita expenditure

7. Social determinants services

(a) Community infrastructure (water, environment, housing)

(b) Community support (education, justice, employment, social services)

(c) Political structure (levels of influence and participation)

(d) Culture and language usage

(e) Racism

(f) Historical and intergenerational trauma

8. Surveillance and monitoring systems, including management and use of information by providers and communities

(a) Availability of data and indicators at different levels of governments across all the services framework domains

(b) Epidemiologic trajectories/timing between initial program funding, services establishment and staff recruitment, program impact (e.g. smoking changes), health outcomes (e.g. changes in smoking related conditions), publication of data on outcomes

(c) Intervention process and outcome measures and timing

(d) Early warning systems

9. Services evaluation

(a) National and state/regional/provincial analyses of specific services

(b) Continuous quality Improvement (CQI) and management processes for use of information e.g., mid-year and annual reviews

(c) Data quality and improved Indigenous identification

(d) Data sharing across jurisdictions

(e) Burden of disease analyses

(f) Satellite and/or outreach services needs assessment

(g) Public versus localised data release protocols and governance

(h) Policy research needs

10. Community self-determination and empowerment measures and services

(a) Nationally agreed policies on data principles

(b) State/regional/provincial data collection, management and use to support community needs

(c) Community data collection, management and use to identify and address local issues at the local level

(d) Individual and family autonomy and support for local knowledge through local data including the role of language translators

(e) Obtaining input from families and community leaders/elders

11. Feedback mechanisms to ensure the framework is dynamic and evolving 
quirement for measurement of need, as estimated by levels of health conditions that are amenable to services (Potentially Preventable Admissions and Potentially Preventable Deaths) as a primary measure of unmet need for services for and by Indigenous people [7]. It highlights the critical issue of access to services, across the spectrum of Indigenous-specific services and general services for the population, and on preferences for, and barriers to, access to those services. It also describes the measurement needs for priority specific service types, both mainstream and traditional healing and medicines, and equity and adequacy in the provision of the workforce for health services as well as ensuring that the workforce and these services are culturally competent and safe for Indigenous peoples [7]. Most of the health measures of Indigenous populations have focussed on ill health including morbidity and mortality with less focus on wellbeing and factors that impact on Indigenous wellbeing measures. In addition, the links between health outcomes and the impact of colonization, racism have not been systemically introduced into the development of health policy that would otherwise consider the impact that culture, language and connection to land can have on the health and well-being of Indigenous peoples. In the Framework, we have addressed the service measures that need to be examined but note that all of these measures will work together in producing policy, program and service implementation.

As all governments struggle with budgetary constraints, especially on health services, it is necessary to examine cost/benefit ratios for different components of health expenditure; but in so doing it is important to consider that cost of service delivery in per capita terms is likely to be higher in remote areas where Indigenous people live [32]. Reduction in Indigenous health services in these areas will likely increase the inequality in health outcomes. Measurement of expenditure is an essential component as is expenditure in relation to need and cost of service delivery in different locations and, most importantly, equity in expenditure for Indigenous and non-Indigenous populations in relation to both need and cost. However, measurements should not overlook the unfair health inequities and the concurrent and historic contextual factors that have resulted in these disparities. It is essential that social determinants such as housing, education, employment and contact with the justice system become essential parts of the measurements. By examining the social determinants in relation to statistics about Indigenous health, it will become apparent that a focus on costs of service delivery alone is insufficient to close the gap in health outcomes. An examination of community infrastructure and services that take culture, language, land and spirituality into consideration are essential to improving health outcomes, promoting revitalization of culture and reducing the systemic racism faced by Indigenous people in Australia, Canada, New Zealand, and the United States.

Evaluation mechanisms, and particularly quality control and health service improvements, are another key aspect of the Framework. Through the use of evaluation mechanisms, the quality of health services provided to Indigenous people can be examined from beginning of and service delivery to the achievement of the desired outcomes [3]. However, statistics alone will not ensure that the funding and service inputs will reduce Indigenous health gaps, and it is necessary to ensure that there is always true collaboration with Indigenous peoples during the creation, development, implementation and funding of services to ensure they continue to meet the needs of the Indigenous population as well as being diverse, culturally sensitive and relevant to communities.

Finally, and importantly, community self determination and empowerment, which have been shown to have an important influence on both service access and effectiveness, are fundamental. Self-determination will lead to true self-governance. It is important to measure how much control (self-determination) that Indigenous communities have over the health services provided in their area. By measuring self-determination, governments can work toward the provision of increased selfdetermination and self-governance, which have been recognised as being important in closing the gap on health outcomes [33-35].

Importantly analyses of Indigenous data should be undertaken in full collaboration with Indigenous peoples, organizations and/or groups that will be affected by the analyses. For the United Nations and the World Health Organization, an examination of health data that focuses on Indigenous-specific data (where available) will provide a baseline of potential programs and services that can be outlined for countries with Indigenous people around the globe. For national and state/regional/provincial governments, an examination of the health data that does not just compare Indigenous peoples to non-Indigenous people will assist with providing strengths-based measures and can lead to improved relations between governments and Indigenous people. This may in turn lead to a fuller examination of health services for Indigenous people. It should also be rec- 
ognized that any analyses of Indigenous data needs to be done through consultation and genuine partnership with Indigenous people and national Indigenous organizations to ensure that health services move toward a positive, culturally-competent change in health services. It is also necessary to recognize the rights and interests of Indigenous peoples in relation to the collection, control and use of the data about themselves. The quality of both the collection of Indigenous health data and use of the data will be improved through consultation and genuine partnership with Indigenous people and national Indigenous organizations. This will ensure that health services have access to reliable data that Indigenous people have trust in to initiate positive, culturally-competent changes in health services.

\section{Conclusions}

The framework presented in this paper was designed to provide direction for health data measurement and analysis that will lead to substantive changes in services for Indigenous people. The health indicators included in the framework focus on providing accuracy and completeness of Indigenous data and address neglected topics such as mental health, social and emotional well-being, culture and wellness. Health services for Indigenous people must be based on accurate data that reflect the realities of the people they serve. The expectation for this framework is that international, national, state/regional/provincial and local governments will examine the components together with their Indigenous counterparts and use the information to address service needs. Central to the framework is ensuring that services delivered to Indigenous peoples have the necessary community input and are made safe and culturally appropriate in terms of systems and workforce This applies to existing services and to future models of service delivery.

Some elements of the framework may be more important to some governments, while other components may be more important to Indigenous communities. This framework, therefore, should be considered as a living document that will develop further through collaboration with Indigenous peoples in Australia, Canada, New Zealand and the United States. Indigenous communities and governments need to focus on implementing the framework for existing and new services. Its implementation should be monitored and reviewed on an ongoing basis to ensure relevant community input. A starting point would be to focus on the implementation of the framework to address issues of social and emotional wellbeing including suicide. Lessons learnt from these programs through case studies can help inform the roll out of the framework to other important services needed by the communities. Positive change will only occur when all levels of government work together with Indigenous peoples to design services that eliminate health disparities and improve Indigenous health and well-being.

As countries work to close the gap in health outcomes between Indigenous and non-Indigenous populations, it is time to rethink how official statistics and health data are collected with a much greater focus on the collection and use of data on the need for, availability, and effectiveness of health services.

\section{References}

[1] Ring I, Brown N. The health status of indigenous peoples and others. BMJ (Clinical research ed). 2003; 327(7412): 404-5.

[2] Anderson I, Robson B, Connolly M, Al-Yaman F, Bjertness E, King A, et al. Indigenous and tribal peoples' health (The Lancet-Lowitja Institute Global Collaboration): A population study. The Lancet. 2016; 388(10040): 131-57.

[3] Australian Institute of Health and Welfare. Timing impact assessment of COAG Closing the Gap targets: Child mortality. Canberra; 2014

[4] Australian Institute of Health and Welfare. Aboriginal and Torres Strait Islander Health Performance Framework 2010: detailed analyses. Canberra; 2010.

[5] Australian Institute of Health and Welfare. Aboriginal and Torres Strait Islander health performance framework 2017: supplementary online tables Canberra: Australian Institute of Health and Welfare; 2017. Available from: https://www.aihw. gov.au/reports/indigenous-health-welfare/health-performance framework/data.

[6] Australian Institute of Health and Welfare. Size and sources of the Indigenous health gap. Canberra: Australian Institute of Health and Welfare; 2018

[7] Australian Institute of Health and Welfare. Access to primary health care relative to need for Indigenous Australians. Canberra; 2014.

[8] National Prevention Council. National Prevention Strategy. Washington, DC: U.S. Department of Health and Human Services - Office of the Surgeon General; 2011.

[9] First Nations Information Governance Centre. National Report of the First Nations Regional Health Survey Phase 3: Volume Two. Ottawa, Ontario: First Nations Information Governance Centre; 2018 July 25, 2018.

[10] Long D, Dickason OE. Visions of the Heart: Canadian Aboriginal Issues, 3rd Edition. Toronto, Ontario: Oxford University Press; 2011.

[11] Waldram JB, Herring DA, Young TK. Aboriginal Health in Canada: Historical, Cultural and Epidemiological Perspectives; 2nd Edition. Toronto, Ontario, Canada: University of Toronto Press; 2006. 367 p.

[12] Ellison-Loschmann L. Improving access to health care among new zealand's maori population. American Journal of Public Health. 2006; 96(4): 612-8. 
[13] Sutherland M, Maar M, Freel S. Improving Access to Quality and Culturally Safe Health Care for Aboriginal Communities in Canada. Dignitas International; 2014.

[14] Australian Institute of Health and Welfare. Child protection Australia 2016-17. Canberra; 2018.

[15] Australian Institute of Health and Welfare. Aboriginal and Torres Strait Islander Stolen Generations and descendants: numbers, demographic characteristics and selected outcomes. Canberra; 2018.

[16] Walls M, Whitbeck L. The intergenerational effects of relocation policies on indigenous families. Journal of Family Issues. 2012; 33(9): 1272-93.

[17] National Native Network. Keep It Sacred: Tribal BRFSS Toolkit Washington, DC: National Native Network; 2017. Available from: http://keepitsacred.itcmi.org/resources/tribal -brfss-toolkit/.

[18] Chandler M, Lalonde C. Cultural continuity as a hedge against suicide in Canada's First Nations. Transcultural Psychiatry. 1998; 35(2): 191-219.

[19] Marles E, Frame C, Royce M. The aboriginal medical service redfern - improving access to primary care for over 40 years. Australian Indigenous Health Bulletin. 2012; 12(3): 433-6.

[20] Boyer Y. Moving Aboriginal health Forward: Discarding Canada's Legal Barriers. Saskatoon, Saskatchewan: Purich Publishing Inc.; 2014. 223 p.

[21] Wilson K. Therapeutic landscapes and First Nations peoples: an exploration of culture, health and place. Health \& Place. 2003; 9: 83-93.

[22] Loppie C, Wien F. Health Inequalities and Social Determinants of Aboriginal Peoples' Health. Victoria, BC: National Collaborating Centre for Aboriginal Health; 2009.

[23] Rhoades E. The indian health service and traditional indian medicine. AMA Journal of Ethics - Virtual Mentor. 2009; 11(10): 793-8.

[24] IHS Director recognizes traditional healing clinic with public health leadership award [press release]. Washington, DC: USDHHS Indian Health Service2011.

[25] Leininger M. Culture care theory: A major contribution to advance transcultural nursing knowledge and practices. Journal of Transcultural Nursing. 2002; 13(3): 189-92.
[26] Australian Health Ministers' Advisory Council. Cultural Respect Framework for Aboriginal torres Strait Islander Health 2016-2026. In: Council AHMA, editor. Canberra2016.

[27] Adams J. Building Cultural Competence: the Medical Council's Direction. The New Zealand Medical Council's approach to cultural competence. Best Practice Journal. 2011; 2011: 18-21.

[28] Walker R, Cromarty H, Linkewich B, Semple D, St. PierreHansen N, Kelly L. Achieving Cultural Integration in Health Services: Design of Comprehensive Hospital Model for Traditional Healing, Medicines, Foods and Supports. Journal of Aboriginal Health. 2010 (January 2010).

[29] De Leeuw S, Parkes M, Thien D. Questioning medicine's discipline: the arts of emotions in undergraduate medical education. Emotion, Space and Society. 2014; 11(43-51).

[30] Health Council of Canada. The Health Status of Canada's First Nations, Metis and Inuit Peoples: A background paper to accompany Health Care Renewal in Canada: Accelerating Change (January 2005). Toronto, Ontario: Health Council of Canada; 2005. Contract No.: Report.

[31] Health Council of Canada. Empathy, dignity, and respect: Creating cultural safety for Aboriginal people in urban health care. Toronto, Ontario; 2012.

[32] Lavoie J, Forget E. A Financial Analysis of the Current and Prospective Health Care Expenditures for First Nations in Manitoba. Winnipeg, Manitoba: Intergovernmental Committe on First Nations Health; 2006.

[33] Companion M. Embracing Autonomy: The Impact of SocioCultural and Political Factors on Tribal Health Care Managment Levels [dissertation]. Tucson, Arizona: University of Arizona; 2003.

[34] Lavoie J. Governed by Contracts: The Development of Indigenous Primary Health Services in Canada, Australia and New Zealand. Winnipeg, MB; 2004 2003. Report No. ISBN 1-8950034-09-4 Contract No. ISBN 1-8950034-09-4.

[35] Mashford-Pringle A. Is There Self-Determination in Canada's First Nations Communities? Journal of Northern Studies. 2016; 10(2): 107-22. 